\title{
Fatores associados à não realização do exame de Papanicolaou: estudo transversal de base populacional em duas capitais brasileiras ${ }^{1}$
}

\author{
Factors associated with non-participation in screening for cervical cancer: \\ population-based cross-sectional study in two Brazilian capitals
}

Autor: Luís Felipe Leite Martins²

Orientadores: Joaquim Gonçalves Valente ${ }^{3}$ e Luiz Claudio Santos Thuler ${ }^{4}$

O câncer do colo do útero inicia-se a partir de uma lesão pré-invasiva, curável em até $100 \%$ dos casos, que geralmente progride lentamente, por anos, antes de atingir o estágio invasor da doença, quando a cura se torna mais difícil, quando não, impossível. A abordagem mais efetiva para o controle do câncer do colo do útero continua sendo o rastreamento por meio do exame preventivo de Papanicolaou. O objetivo deste estudo foi identificar fatores associados à não realização do exame de Papanicolaou em mulheres de 25 a 59 anos nos últimos três anos anteriores à pesquisa, nos municípios de Fortaleza e Rio de Janeiro. Para cada localidade foi utilizado o delineamento transversal, de base populacional, com amostragem por conglomerados com dois estágios de seleção e auto-ponderada. Os dados foram analisados por regressão de Poisson obedecendo a um modelo hierárquico previamente determinado. O percentual de mulheres não submetidas ao exame de Papanicolaou nos três anos anteriores à pesquisa, em Fortaleza e no Rio de Janeiro, foi de 19,1\% (IC95\%: 16,1-22,1) e 16,5\% (IC95\%: 14,118,9), respectivamente. Mulheres de baixa escolaridade e menor renda per capita, de maior idade, não casadas, não submetidas à mamografia, ao exame clínico das mamas e aos exames de glicemia e colesterolemia foram as que apresentaram as maiores razões de prevalências para a não realização do exame de Papanicolaou, em ambas as localidades. Além disso, as fumantes foram menos submetidas ao exame em relação às demais mulheres, havendo uma diferença estatisticamente significativa somente no Rio de Janeiro. Finalmente, as informações aqui apresentadas apontam para a necessidade de intervenção em um grupo específico de mulheres. Deve-se priorizar atividades de educação para o diagnóstico precoce e rastreamento em mulheres sintomáticas e assintomáticas, respectivamente, além da garantia de acesso aos métodos de diagnóstico e tratamento adequados.

Palavras-chaves: Neoplasias do colo uterino; Esfregaço vaginal; Prevenção e controle; Estudos transversais; Brasil.

\footnotetext{
${ }^{1}$ Dissertação de mestrado apresentada ao curso de pós-graduação em Saúde Coletiva (Epidemiologia) do Instituto de Medicina Social (IMS) da Universidade do Estado do Rio de Janeiro (UERJ). Rio de Janeiro, dezembro de 2004.

${ }^{2}$ Mestre pelo curso de pós-graduação em Saúde Coletiva (Epidemiologia) do Instituto de Medicina Social (IMS) da Universidade do Estado do Rio de Janeiro (UERJ) e Analista de Programa de Controle de Câncer da Divisão de Epidemiologia/Coordenação de Prevenção e Vigilância do Instituto Nacional de Câncer (INCA).

${ }^{3}$ Pesquisador do Departamento de Epidemiologia e Métodos Quantitativos em Saúde da Escola Nacional de Saúde Pública (ENSP) da Fundação Oswaldo Cruz (FIOCRUZ) e Professor Adjunto do Departamento de Epidemiologia do Instituto de Medicina Social (IMS) da Universidade do Estado do Rio de Janeiro (UERJ).

${ }^{4}$ Médico Epidemiologista do Instituto Nacional de Câncer (INCA) e Professor Adjunto da Universidade Federal do Estado do Rio de Janeiro (UNIRIO). Endereço para Correspondência: Luís Felipe Leite Martins - Rua dos Inválidos 212 - 3o andar - Centro - 20.231-048 - Rio de Janeiro - RJ.

E-mail:1fmartins@inca.gov.br
} 